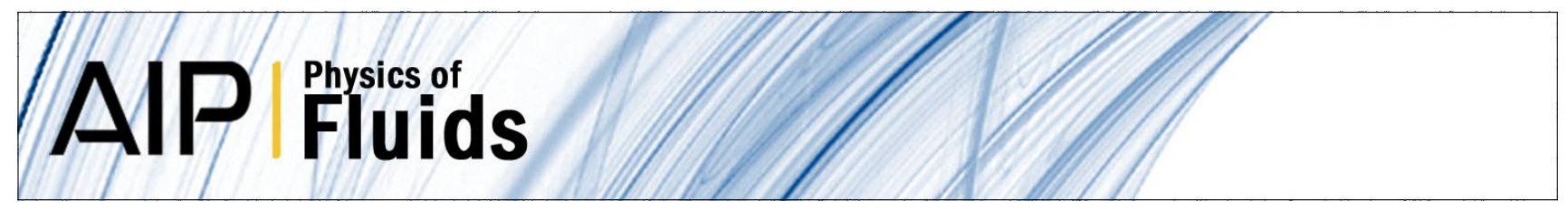

\title{
Fluid velocity fluctuations in a collision of a sphere with a wall
}

J. Rafael Pacheco, Angel Ruiz-Angulo, Roberto Zenit, and Roberto Verzicco

Citation: Phys. Fluids 23, 063301 (2011); doi: 10.1063/1.3598313

View online: http://dx.doi.org/10.1063/1.3598313

View Table of Contents: http://pof.aip.org/resource/1/PHFLE6/v23/i6

Published by the American Institute of Physics.

\section{Related Articles}

A study of thermal counterflow using particle tracking velocimetry

Phys. Fluids 23, 107102 (2011)

Particle accumulation on periodic orbits by repeated free surface collisions

Phys. Fluids 23, 072106 (2011)

Drag force of a particle moving axisymmetrically in open or closed cavities

J. Chem. Phys. 135, 014904 (2011)

Modal and non-modal stability of particle-laden channel flow

Phys. Fluids 23, 064110 (2011)

The suspension balance model revisited

Phys. Fluids 23, 043304 (2011)

\section{Additional information on Phys. Fluids}

Journal Homepage: http://pof.aip.org/

Journal Information: http://pof.aip.org/about/about_the_journal

Top downloads: http://pof.aip.org/features/most_downloaded

Information for Authors: http://pof.aip.org/authors

\section{ADVERTISEMENT}

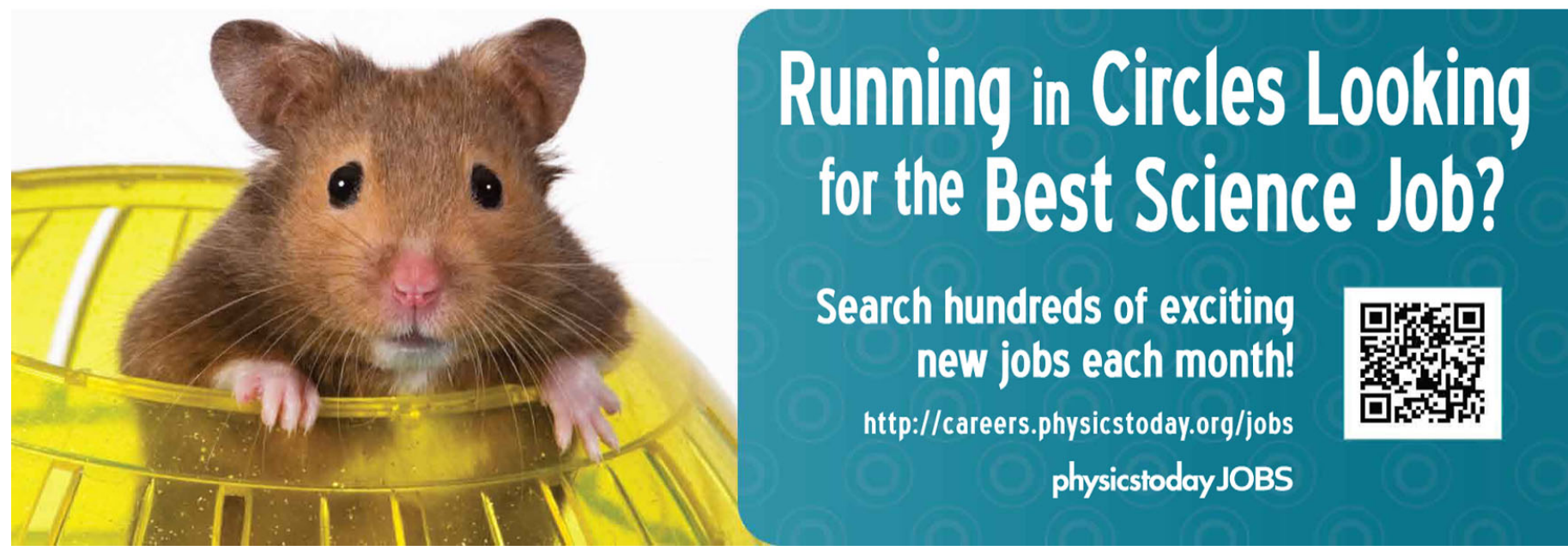




\title{
Fluid velocity fluctuations in a collision of a sphere with a wall
}

\author{
J. Rafael Pacheco, ${ }^{1, a)}$ Angel Ruiz-Angulo, ${ }^{2}$ Roberto Zenit, ${ }^{2}$ and Roberto Verzicco ${ }^{3}$ \\ ${ }^{1}$ School of Mathematical and Statistical Sciences, Arizona State University, Tempe, Arizona 85287, USA and \\ Environmental Fluid Dynamics Laboratories, Department of Civil Engineering and Geological Sciences, \\ The University of Notre Dame, South Bend, Indiana 46556, USA \\ ${ }^{2}$ Instituto de Investigaciones en Materiales, Universidad Nacional Autónoma de México, \\ México D.F.04510, México \\ ${ }^{3}$ Dipartimento di Ingegneria Meccanica, Universita' di Roma “Tor Vergata," Via del Politecnico 1, \\ 00133, Roma, Italy and PoF, University of Twente, 7500 AE Enschede, The Netherlands
}

(Received 29 November 2010; accepted 18 May 2011; published online 23 June 2011)

\begin{abstract}
We report on the results of a combined experimental and numerical study on the fluid motion generated by the controlled approach and arrest of a solid sphere moving towards a solid wall at moderate Reynolds number. The experiments are performed in a small tank filled with water for a range of Reynolds numbers for which the flow remains axisymmetric. The fluid agitation of the fluid related to the kinetic energy is obtained as function of time in the experiment in a volume located around the impact point. The same quantities are obtained from the numerical simulations for the same volume of integration as in the experiments and also for the entire volume of the container. As shown in previous studies, this flow is characterized by a vortex ring, initially in the wake of the sphere, that spreads radially along the wall, generating secondary vorticity of opposite sign at the sphere surface and wall. It is also observed that before the impact, the kinetic energy increases sharply for a small period of time and then decreases gradually as the fluid motion dies out. The measure of the relative agitation of the collision is found to increase weakly with the Reynolds number $R e$. The close agreement between the numerics and experiments is indicative of the robustness of the results. These results may be useful in light of a potential modelling of particle-laden flows. Movies illustrating the spatio-temporal dynamics are provided with the online version of this paper. (C) 2011 American Institute of Physics. [doi:10.1063/1.3598313]
\end{abstract}

\section{INTRODUCTION}

Particulate two-phase flows are prominent in industrial applications and natural phenomena, but despite its importance, a thorough understanding is still deficient. Coal-based energy systems such as pulverized coal boilers and gasifiers are of current interest in industry due to the increase in energy demand. In nature, the movement of sediment bedload due to flash-flooding on alluvial fans is also important because it may place many communities at high risk during intense and prolonged rainfall.

Particulate two-phase flows have turbulent-like behavior at lower Reynolds numbers than those observed in singlephase turbulent flows. This characteristic makes two-phase flows very attractive in industrial applications. ${ }^{1}$ Since the interstitial fluid must move around the inclusions that form the particulate phase, a velocity disturbance (agitation) naturally arises in the continuous phase. Integral measures (such as impulse, circulation, and kinetic energy) have been used as diagnostic tools to study vortical and turbulent flows. ${ }^{2}$ However, in the field of dispersed multiphase flows, integral measures have only been applied in a few instances (see Ref. 3 and references therein), and closure relations that can be used to predict fluctuations from first principles (without questionable assumptions) are scarce. There are notable exceptions, e.g., the case of bubbly liquids at high Reynolds and at low Weber numbers ${ }^{4,5}$ and the case of low Reynolds

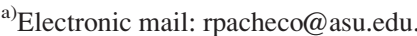

number suspensions, for which models have been proposed to predict the hydrodynamic fluctuations for both sedimenting particles $^{6}$ and simple shear flows. ${ }^{7}$

Perhaps the simplest way to view the agitation phenomenon is by relating it to the added mass, because the added mass determines the necessary work done to change the agitation associated with the fluid motion. ${ }^{8-10}$ The agitation is important in situations of flows with non-dilute particle loading. ${ }^{11}$ Transfer processes, such as dust resuspension (for particles), heat transfer (by vapor bubbles), or interfacial gas transfer across a free surface (e.g., bubbles) predominately arise from the significant agitation afforded by the movement of discrete elements close to boundaries. ${ }^{12-14}$ The kinematic blocking motion caused by a boundary (such as a rigid wall or free surface) inhibits the effects of external turbulence (for instance, by convection or the ambient flow) moving fluid near boundaries. Viscous effects further reduce these effects by creating boundary layers which are typically much larger than the discrete elements. The effect of discrete elements striking or moving near to boundaries creates boundary layers, for instance by sand particles, which are much thinner and flows faster than those created by external motions. This is why sand particles enhance dust resuspen$\operatorname{sion}^{14-16}$ and vapor bubbles enhance heat transfer. ${ }^{3,12,17}$

Practical engineering models of boundary transfer processes (such as heat transfer, dust resuspension, or dilute gas fluidised beds) require closure relationships that relate the motion of elements close to boundaries with a degree of agitation of the fluid. Presently, most dispersed multiphase flow 
models are based on the kinetic theory of the discrete and continuous phases, in addition to Reynolds and phase averaged momentum equations which are analogous to $k-\varepsilon$ models of turbulence, techniques common in engineering. ${ }^{2}$ A practical closure relationship requires single (integral) measures that can be incorporated into coupled two-phase kinetic models. There is a practical and well-established methodology for interior flows, away from boundaries, but a major pressing question is what conditions need to be applied near boundaries. In this paper, we undertake detailed calculations on the fluid motion around a sphere colliding with a wall immersed in a viscous fluid and establish a new methodology utilizing integral measures that in part permit practical closure relationships near walls to be prescribed.

The problem combines many subjects of interest in fluid mechanics: the detachment of a wake due to the unsteady motion of an object, ${ }^{18}$ the interaction a vortex ring with a sphere and a wall, ${ }^{19}$ and the rebound of a particle colliding immersed in a liquid. ${ }^{15,16,20,21}$ The numerical methods and experimental techniques used in this paper are summarized in Sec. II, all of which have already been used in the analysis of different but related problems, ${ }^{20-23}$ and only the salient aspects are presented. The results from numerical simulations and experiments are analyzed in Sec. III which includes a definition of the fluid agitation. Summary and conclusions are presented in Sec. IV.

\section{NUMERICAL SCHEME AND THE EXPERIMENTAL SETUP}

Consider the flow in a completely filled cylinder of fluid with kinematic viscosity $\nu$ of radius $R$ and height $H$. The walls are stationary and the flow is driven by the motion of the sphere, which is impulsively started from rest at constant speed normal to the bottom wall and stops after traveling a distance $h$. To non-dimensionalize the system, the diameter of the sphere $D$ is used as the length scale, the velocity scale is the constant velocity of the sphere before impact $U_{p}$, and the time scale is the inertial time $D / U_{p}$.

In cylindrical coordinates $(r, \theta, z)$, the non-dimensional velocity vector, pressure, and time are denoted by $\mathbf{u}=$ $(u, v, w), p$, and $t$, respectively. The system is governed by four non-dimensional parameters, three geometric and one dynamic: the radius of the container $\rho=R / D$, the aspect ratio $\Gamma=H / R$, the traveling distance $\eta=h / D$, and the Reynolds number $R e=U_{p} D / \nu$. A schematic of the flow geometry, with an inset showing the azimuthal vorticity at $R e=400, \rho=10, \Gamma=1.5$, and $\eta=8$, is given in Fig. 1 .

The governing equations are the (non-dimensional) incompressible Navier-Stokes equations

$$
\partial \boldsymbol{u} / \partial t+(\boldsymbol{u} \cdot \nabla) \boldsymbol{u}=-\nabla p+R e^{-1} \nabla^{2} \boldsymbol{u}, \quad \nabla \cdot \boldsymbol{u}=0 .
$$

The boundary conditions for the velocity field are stress-free at the top and no-slip on the side/bottom walls. On the sphere, $u$ and $v$ are zero for all times and $w=-1$ for $t<0$ (prior to impact) and $w=0$ for $t \geq 0$ (perfect inelastic collision).

Equation (1) is solved using a fractional-step scheme. The discretization of both viscous and advective terms is per-

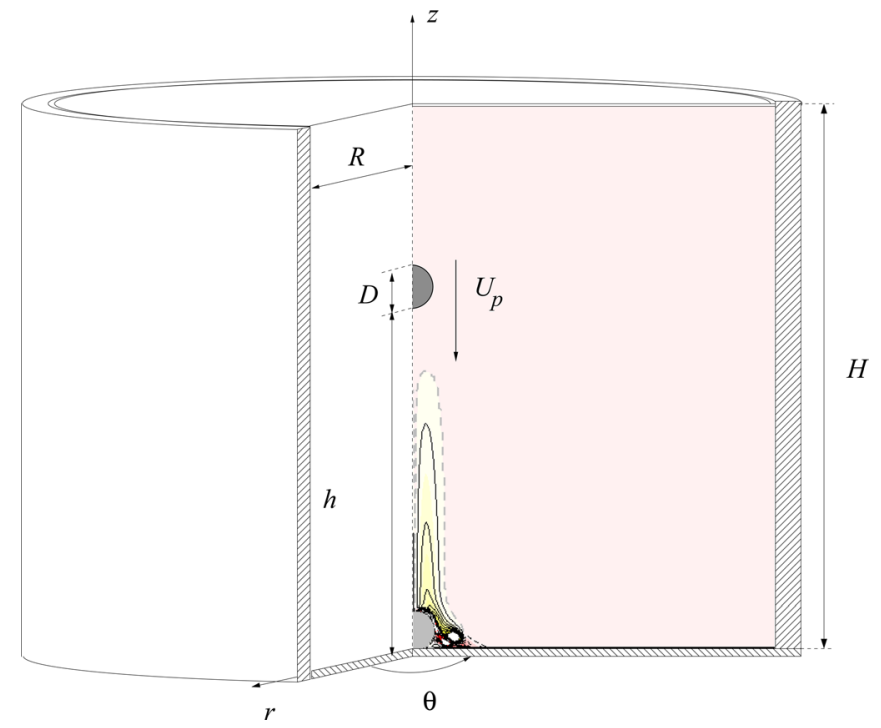

FIG. 1. (Color online) Schematic of the flow apparatus. The inset shows the initial position of the sphere and the vorticity contour of azimuthal vorticity $\omega_{\theta}$ (from numerical simulations) for $t>0$ after the sphere has touched the wall at $R e=400, \rho=10, \Gamma=1.5$, and $\eta=8$.

formed by second-order-accurate central finite-difference approximations. The elliptic equation, necessary to enforce incompressibility, is solved directly using trigonometric expansions in the azimuthal direction and the tensor-product method $^{24}$ for the other two directions. Temporal evolution is via a third-order Runge-Kutta scheme which calculates the nonlinear terms explicitly and the viscous terms implicitly. The stability limit due to the explicit treatment of the convective terms is CFL $<\sqrt{3}$, where CFL is the Courant, Friedrichs and Lewy number. ${ }^{25} \mathrm{~A}$ useful feature of this scheme is the possibility to advance in time by a variable time step, without reducing the accuracy or introducing interpolations. We have varied $\delta t$ in all the simulations in this paper such that the local CFL $\leq 1.5$, where CFL $=(|u| / \delta r+|v| /$ $(r \delta \theta)+|w| / \delta z) \delta t$, with the velocity components averaged at the center of each computational cell. The smallest such determined local $\delta t$ is then used for time advancement (see Ref. 26 for more details, including the treatment of the radial axis).

The immersed boundary method (IB) is used in this study to simulate the sphere. The main advantage of using the IB consists in solving flows bounded by arbitrarily complex geometries without resorting to body-conformal grids for which the motion is prescribed, and, therefore, the solution technique essentially has the same ease of use and efficiency as that of simple geometries. The method is second-order in space and this technique has already been implemented in many different scenarios and grid layouts, e.g., laminar and turbulent convection, ${ }^{27-29}$ turbulent flows and particle collision, ${ }^{30-33}$ biological devices, ${ }^{34}$ and bifurcations. ${ }^{35}$ The threedimensional simulations were conducted using the immersed boundary method of Ref. 36 .

Numerical simulations were conducted with different grid sizes to verify the grid-independence results and to test the adequacy of a coarser grid in resolving all the relevant flow scales. We placed several probes in the neighborhood 
(a)

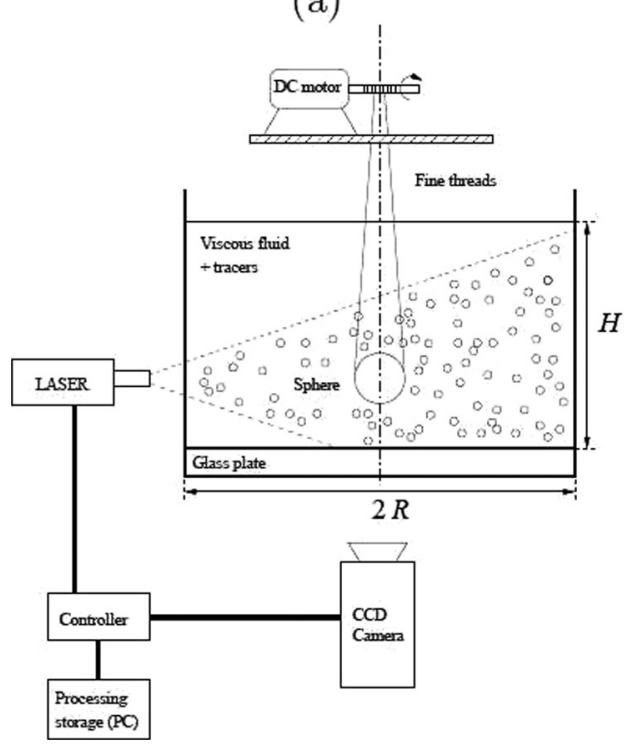

(b)

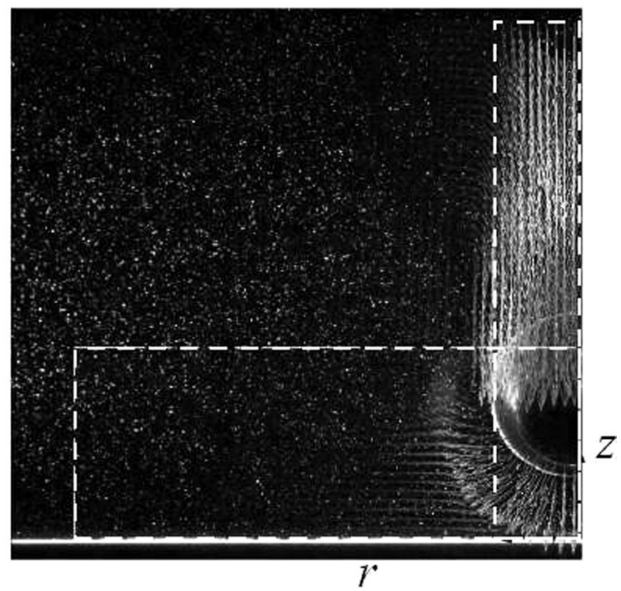

FIG. 2. (a) Experimental apparatus used to generate controlled collisions of a sphere with a wall. (b) Typical PIV image and velocity field at $R e=300$; $D=25.4 \mathrm{~mm}$. The field of view is approximately $75 \times 85 \mathrm{~mm}^{2}(\approx 3 \times 3.3$ dimensionless units). The white dashed lines correspond to the areas used to evaluate the agitation in the experiments. where the collision took place and measured the velocities at different times. We then reduced the grid size using resolutions of up to $n_{\theta} \times n_{r} \times n_{z}=96 \times 301 \times 601$. We find that a grid resolution of $64 \times 151 \times 251$ results in a maximum difference in velocities at the probe locations of less than $1 \%$ compared to the finer grid, and this resolution has been used in most of the runs presented in this paper. To determine the azimuthal symmetry of the flow, we have also performed similar checks as in Refs. 37 and 38 who studied different but related problems of baroclinic instabilities in the presence of rotation and stratification.

The experimental setup of the system used for this study is shown in Fig. 2(a) and consists of a stainless steel sphere of density $7.9 \mathrm{~g} / \mathrm{cm}^{3}$ and diameter $25.4 \mathrm{~mm}$ in water at laboratory conditions $\left(\nu=10.2 \mathrm{~mm}^{2} \mathrm{~s}^{-1}\right)$ with an absolute uncertainty of $\pm 0.1 \mathrm{~mm}^{2} \mathrm{~s}^{-1}$ confined in a rectangular glass container of $2 R \times 2 R \times H=30 \times 30 \times 50 \mathrm{~cm}^{3}(\approx 12 \times 12$ $\times 20$ dimensionless units). The corresponding range of particle Reynolds number ranged between 50 and 400. A thick glass plate was placed at the bottom of the container and the particle release mechanism was placed at the top on the lid of the container where two fine Nylon threads were glued to the particle poles to inhibit rotation. The motion of the particle was controlled by slowly unwinding the threads from the shaft of a computer-controlled DC motor, allowing the particle to touch the bottom wall without any noticeable bouncing after impact.

To visualize and quantify the velocity field around the sphere during the collision process, an ordinary 2-D particle image velocimetry (PIV) system (Dantec Flowmap 1500 model) was used. The flow was illuminated with a pulsed laser sheet of approximately $0.5 \mathrm{~mm}$ of thickness. The laser sheet formed a small angle with the plane made by the two strings attached to the sphere. Images of the laser illuminated plane were obtained with a $1000 \times 1000$ pixel digital camera. The laser and the camera were synchronized by a control unit that allowed the adjustment of the time between frames as well as the time between pairs of frames. The typical time between frames used to calculate the velocities was in the order of $10 \mathrm{~ms}$. The time between pairs of photographs was $160 \mathrm{~ms}$, the highest allowed by the system. The field of view of the camera was approximately $75 \times 85 \mathrm{~mm}^{2}(\approx 3 \times 3.3$ dimensionless units). An adaptive cross-correlation technique was used, with a final interrogation area of $32 \times 32$ pixels and an overlap of $50 \%$ in both directions. Subsequently, a peak validation, moving average and spacial filter routines were applied. The resolution of the optical array was $12.5 \mathrm{pixel} / \mathrm{mm}$. Using the uncertainty protocol of Ref. 39 , the velocity uncertainty was calculated to be about $5.3 \%$. The experimental setup and a typical PIV image are shown in Fig. 2, where the velocity field shown was superimposed on the PIV image. For all cases, the area of the sphere was masked and only half of the flow field was shown and processed, because the sphere blocked the laser light.

For a given experiment, the particle was placed originally at a distance of eight particle diameters from the bottom plate $(\eta=8)$, and the voltage was set to a constant value before energizing the motor. After a very small transient, the sphere began to descend at constant velocity. Since the particle Reynolds number was large, a deceleration of the particle was not observed before it collided with the wall. ${ }^{20}$

\section{RESULTS}

The experimental measurements and most of the numerical simulations were conducted for a range of particle Reynolds numbers $100 \leq R e \leq 400$ and $\eta=8$ to minimize the possibility of a three-dimensional flow around the sphere. ${ }^{19}$ Contour plots of the azimuthal vorticity $\omega_{\theta}$ at $R e=400$, $\rho=10, \Gamma=1.5$, and $\eta=8$ are shown in Fig. 3 and the associated movie 3 shows typical states in this regime. The time origin has been displaced such that $t=0$ corresponds to the time when the sphere makes contact with the solid surface. It can be observed that after the collision, the wake (originally in the back of the sphere) moves forward around the surface of the sphere $(0<t<1)$. At $t \approx 2$, the vortex hits the wall 


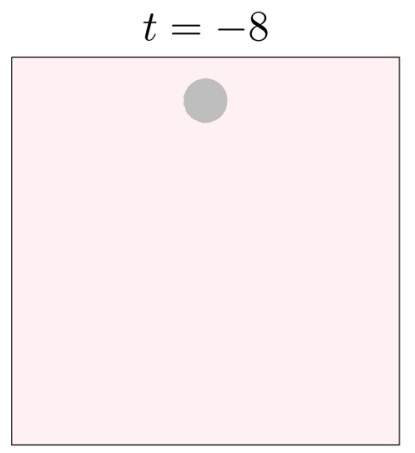

$t=-2$

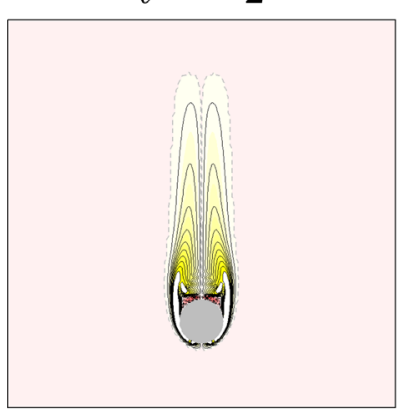

$t=4$
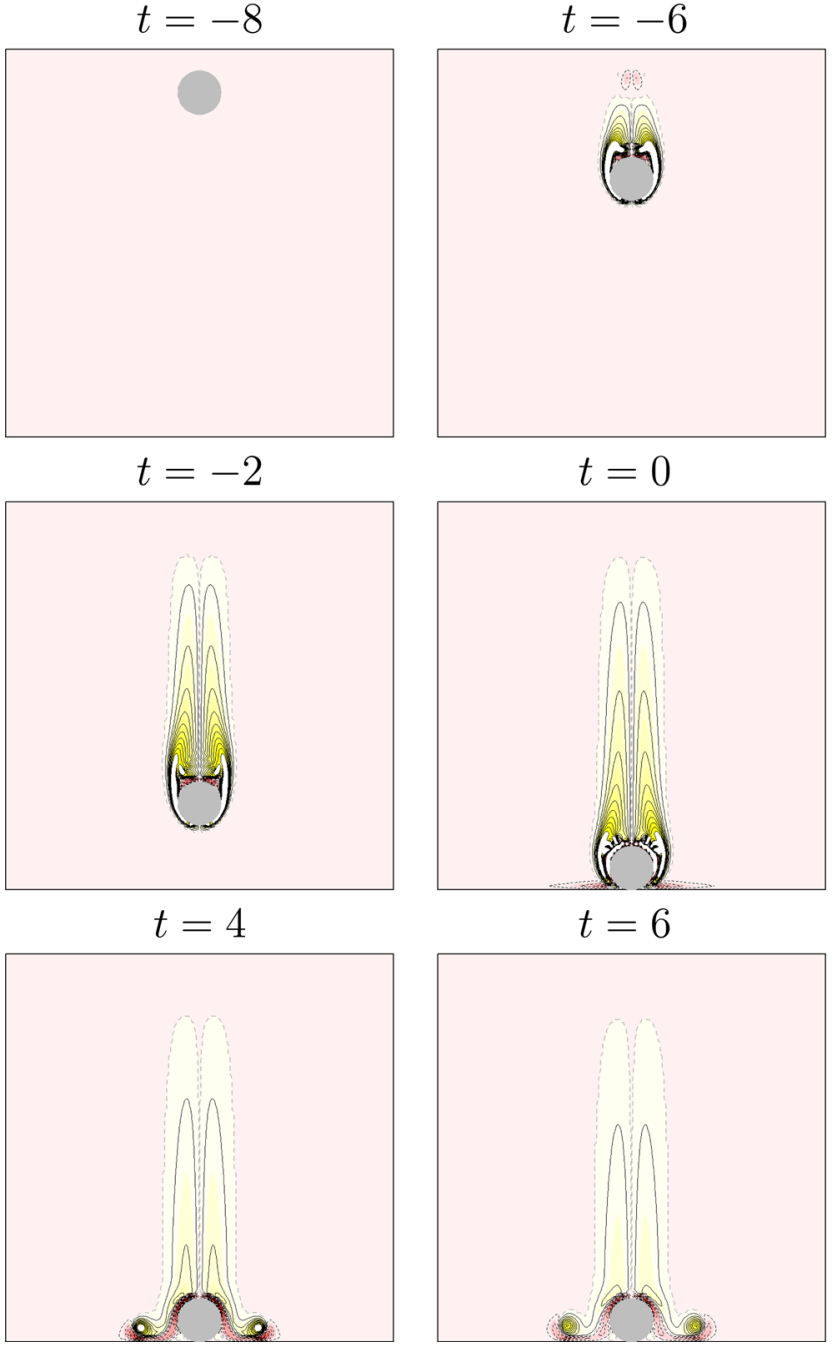

$t=0$

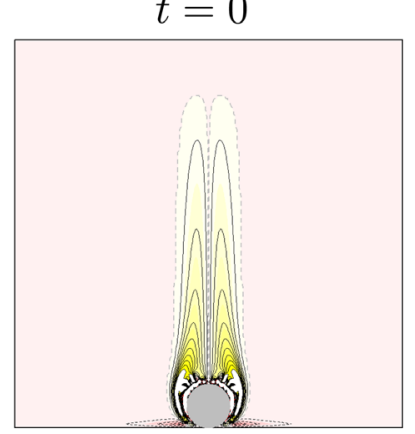

$t=6$

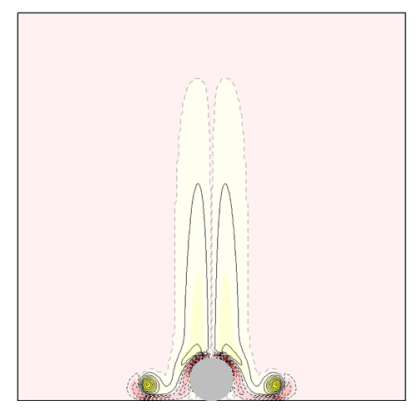

$t=-4$
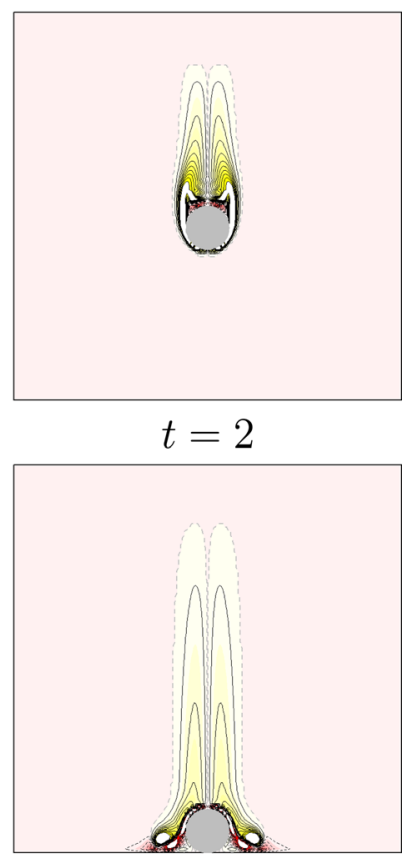

$t=8$

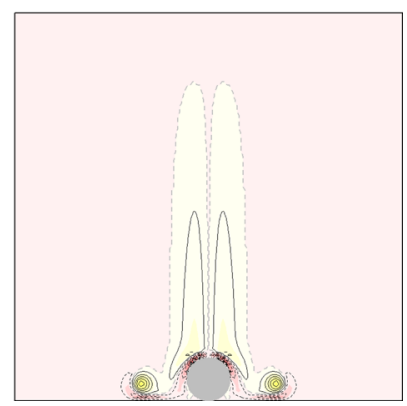

FIG. 3. (Color online) Contours of azimuthal vorticity $\omega_{\theta}$ from numerical simulations at $R e=400, \rho=12, \eta=8$, $\Gamma=1.25$ and various $t$ as indicated. The region shown is $-4.5 \leq r \leq 4.5$ and $0 \leq z \leq 9$. There are 20 positive (-) and negative $(----)$ linearly spaced contour levels in the ranges $\omega_{\theta} \in[-1.5,1.5]$. The zero contour is the grey line. The associated animation is available online as movie 3 (enhanced online). [URL: http://dx.doi.org/10.1063/ 1.3598313.1]. and begins to spread radially and at $t \geq 4$, the center of the ring remains fixed in space and the fluid motion decreases gradually due to the viscous dissipation. The nature of the wake's motion described above is qualitatively similar for other values of Reynolds numbers and is in agreement with other numerical and laboratory studies. ${ }^{16,40,41}$

\section{A. Fluid agitation}

We are interested in quantifying the fluid disturbance caused by the collision of a single particle. A measure of the fluid disturbance can be obtained from the velocity fields by defining an agitation quantity (kinetic energy per unit mass) within a volume of fluid that excludes the volume of the particle, as

$$
A(t)=\int_{V}\left(u^{2}+v^{2}+w^{2}\right) \mathrm{d} V
$$

where $V$ is a control volume over which the agitation is measured and $(u, v, w)$ are the vector components of fluid velocity in the radial, azimuthal, and vertical directions, respectively.

Ideally, the dimensions of the container should be as large as possible, because far from the sphere, the velocity of the fluid is negligible and the walls would have a negligible influence in the flow dynamics. However, in any laboratory experiment, there are limitations in the size of the measuring area (in our case, the field of view of camera in the experiments was approximately $3 \times 3.3$ dimensionless units). We have measured the agitation in the experiments for two different areas of field view and compared these results with those from the Navier-Stokes solver for verification of the correct implementation of the immersed boundary method. Figure 4 shows the evolution of the agitation from Eq. (2) at $R e=400$ and a comparison with the numerical results. The region of integration to determine the agitation shown in Figure 4(a) is from $r=0 \rightarrow 3$ and $z=0 \rightarrow 1$ and that of Fig. 4(b) is from $r=0 \rightarrow 0.5$ and $z=0 \rightarrow 3.3$. The sharp increase in the agitation after the sphere impact shown in Figure 4(a) is due to the fluid motion in the wake of the particle before the collision that was excluded in the area of integration and not by the impact of the sphere. The rise in the agitation shown in Fig. 4(b) is because the sphere enters the domain of integration and accounts for a narrow region that included the wake of the sphere.

In the original quiescent fluid, the flow is generated by the sphere motion, thence the fluid is already agitated before impact. Consequently, to quantify the agitation caused by 
(a)

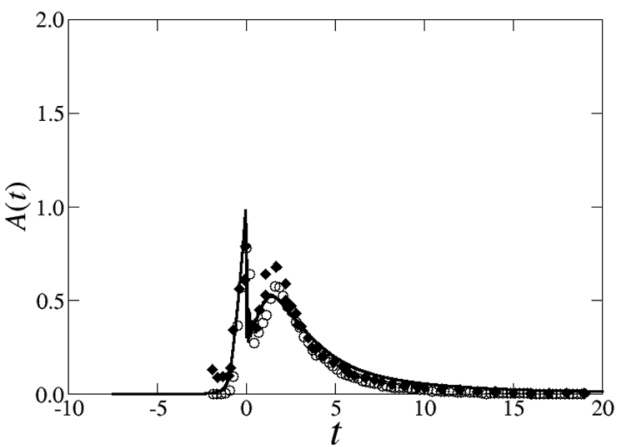

(b)

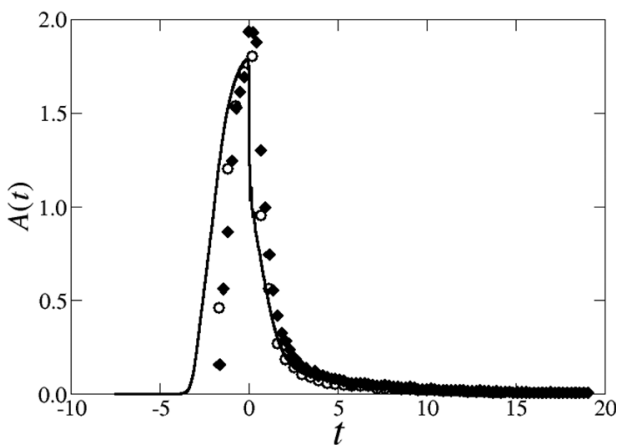

FIG. 4. Fluid agitation at $R e=400$, $\rho=10, \Gamma=1.5$, and $\eta=8$ for two different regions of integration. The results from two different experiments are indicated with symbols ${ }^{\circ}$ and $\bullet$ and the numerical solution with solid line. The regions of integration are from $\theta=0 \rightarrow 2 \pi$ and (a) $r=0 \rightarrow 3$, $z=0 \rightarrow 1$; (b) $r=0 \rightarrow 0.5, z=0 \rightarrow 3$. the motion of the sphere, the entire volume should be considered at all times. If measured in this way, there is no increase in the overall agitation after the sphere touches the wall; instead, it starts to decrease as soon as the body stops. The curves of agitation for different limits of integration (but excluding the particle) are shown in Fig. 5 depicting their asymptotic behavior as the volume of integration is increased. The sharp drop in the agitation (solid line of Fig. 5) at $t=0$ is due to the perfectly inelastic collision of the sphere with the bottom wall. At $t>0$, the energy begins to decay due to viscous dissipation.

The agitation described above includes both the energy due to the motion of the sphere as well as the part due to the impact. To quantify the sole effect of the collision in terms of an agitation measure, from a flow that is both unsteady and varying in space, we require to extract the influence of start-up motion towards steady state and arrest of the sphere on the wall. To this end, we perform the same simulations using an extended axial domain with the lower wall mimicked (see the right side of Fig. 6) where the sphere is stopped at the imaginary bottom boundary, but the fluid is

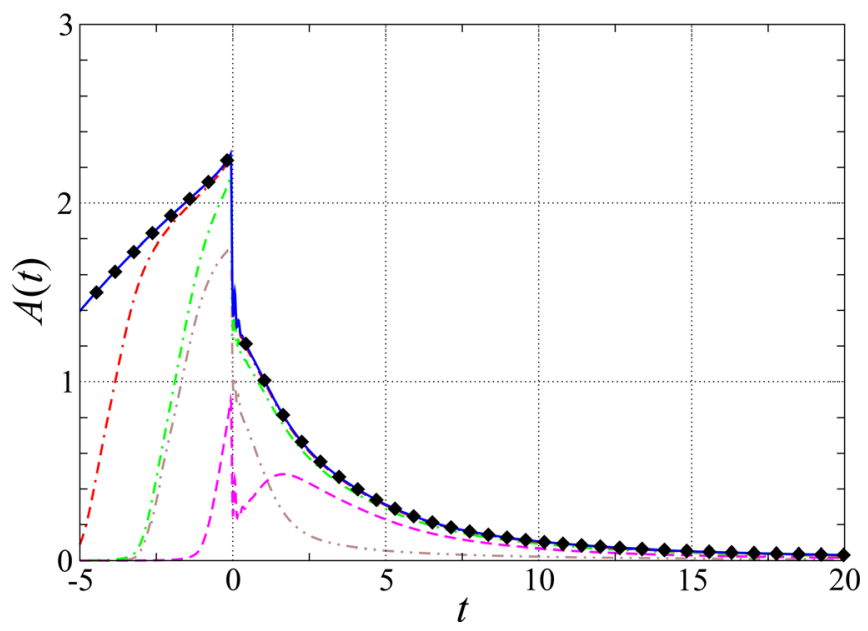

FIG. 5. (Color online) Fluid agitation as a function of the measuring volume. The case shown is a numerical run at $\operatorname{Re}=400, \rho=12, \eta=8$, and $\Gamma=1.25$. The regions of integration are from $\theta=0 \rightarrow 2 \pi$ and $(--)$ $r=0 \rightarrow 3, \quad z=0 \rightarrow 1 ; \quad(-\cdots-) \quad r=0 \rightarrow 0.5, \quad z=0 \rightarrow 3 ; \quad(-\cdot-)$ $r=0 \rightarrow 5, \quad z=0 \rightarrow 8 ; \quad(--\cdot--) \quad r=0 \rightarrow 5, \quad z=0 \rightarrow 8 ; \quad(-)$ $r=0 \rightarrow 7, z=0 \rightarrow 12 ;(\diamond) r=0 \rightarrow 9, z=0 \rightarrow 14$. allowed to flow through. We then monitored the agitation in the extended cylinder within the region delimited by the boundary of the control volume marked by the grey line of Figure 6 (the same volume as that of the standard cylinder) before and after impact, including the vortex ring. As the particle approaches the wall in the standard cylinder, the fluid is squeezed out of the gap between the particle and the wall. This strong shear rate in the gap region is absent in (a) $t=-8$

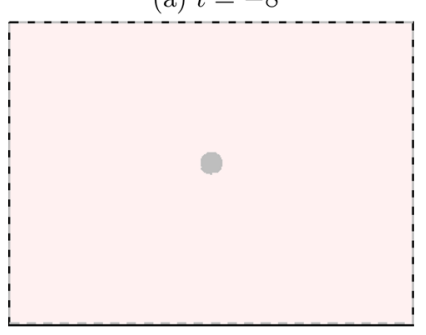

(c) $t=7$

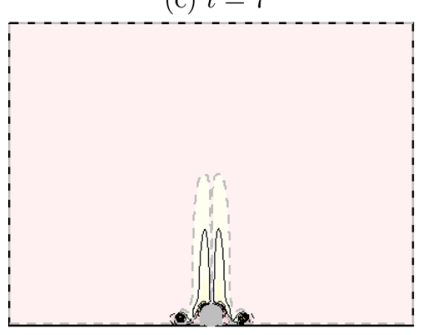

(b) $t=-8$

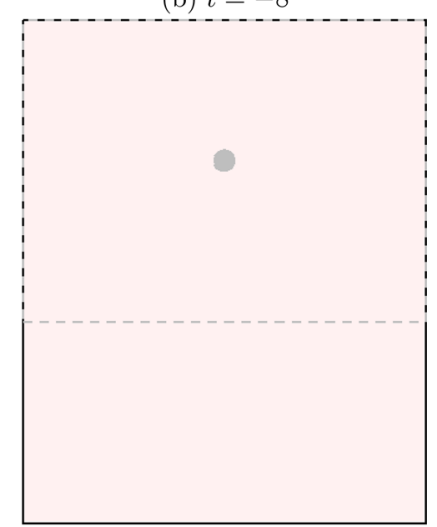

(d) $t=7$

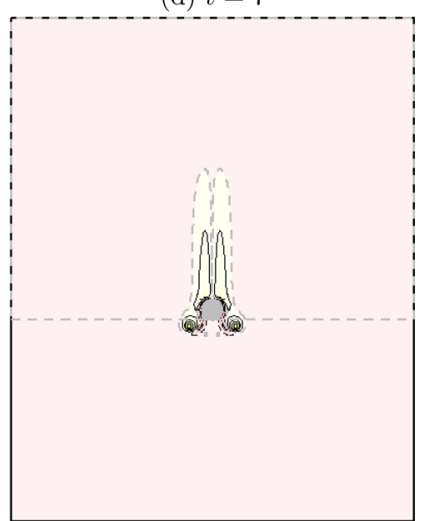

FIG. 6. (Color online) Contours of azimuthal vorticity $\omega_{\theta}$ at $R e=400$, and $t$ as indicated, from numerical simulations. The grey lines in the background are the boundaries of the control volume used to evaluate the agitation. For the standard cylinder (a) and (c), $\rho_{s}=10, \Gamma_{s}=1.5, \eta_{s}=8$ and region of integration is from $r=0 \rightarrow 10$ and $z=0 \rightarrow 15$. For the extended cylinder (b) and (d), $\rho_{e}=10, \Gamma_{e}=2.5, \eta_{e}=18$ and the region of integration is from $r=0 \rightarrow 10$ and $z=10 \rightarrow 25$. There are 20 positive $(-)$ and negative $(----)$ linearly spaced contour levels in the ranges $\omega_{\theta} \in[-1.5,1.5]$. 
(a) $R e=100$

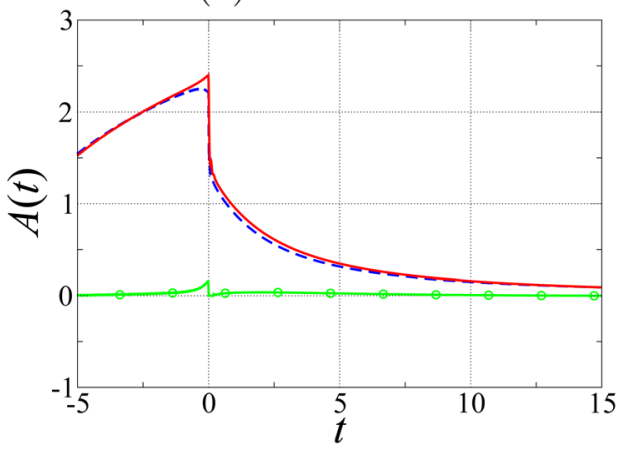

(b) $R e=300$

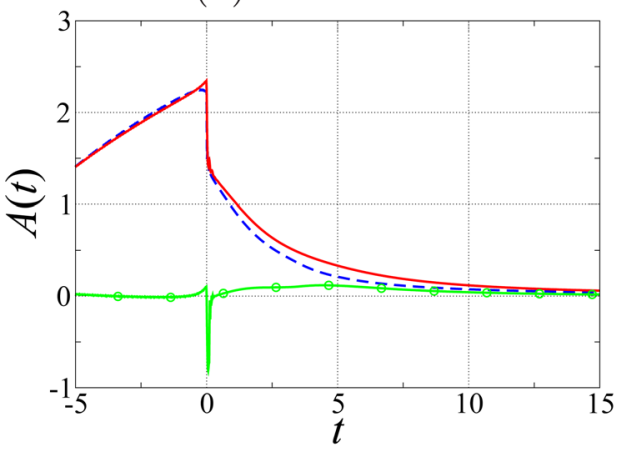

(d) $R e=500$

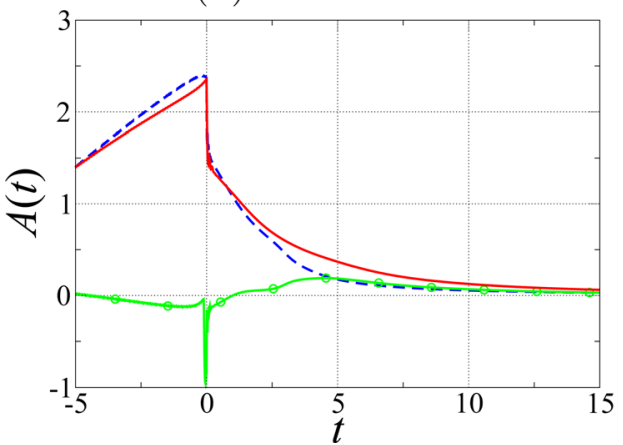

(b) $R e=200$

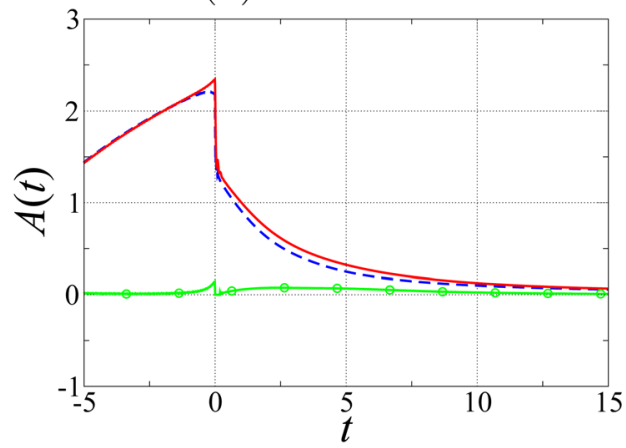

(c) $R e=400$

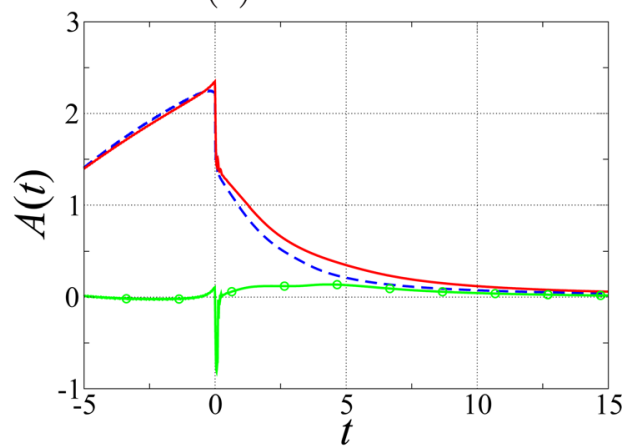

(e) $R e=600$

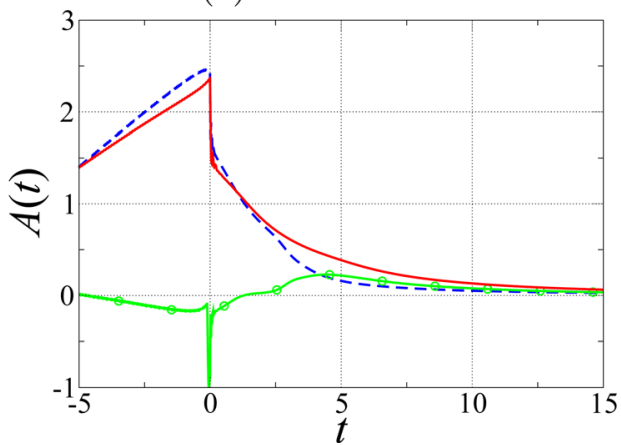

FIG. 7. (Color online) Fluid agitation at $100 \leq R e \leq 600$ from numerical simulations. For the standard cylinder $\eta_{s}=8$, $A_{s} \quad(-)$, for the extended cylinder $\eta_{e}=18, A_{e}(--)$, and the difference $A_{c}$ $\left(-{ }^{\circ}-\right)$. the extended cylinder and thus the sole effect of the collision should be the difference between these evolutions.

If we let the subscripts $s$ and $e$ denote the standard and extended cylinders, respectively, then a quantity with the subscript $c$ implies the difference between the corresponding quantities calculated in the standard cylinder and the extended cylinder, i.e., $(\cdot)_{c}=(\cdot)_{s}-(\cdot)_{e}$. Thus, the agitation due to the collision alone may be written as $A_{c}=A_{s}-A_{e}$. Notice that far away from the walls, the agitation produced by the sphere in both the standard and extended cylinders would be the same, but as the sphere approaches the solidmimicked wall, they would begin to differ.

Figure 6 shows contours of azimuthal vorticity, the control volume, and the location of the spheres for the two configurations at two different times (before and after the sphere stops). The initial time of release of the sphere was at $t=-8$. At $t=7$, the vortex ring in the standard cylinder has collided with the wall, whereas in the extended cylinder, the vortex ring has already crossed the imaginary lower boundary (dashed grey line corresponding to the bottom wall of the standard cylinder). The grids in the region of integration for both cylinders are the same. However, for the extended cylinder, additional grid points extend below the bottom wall compared to the standard cylinder.

The time evolution of the agitation from Eq. (2) for both the standard and extended cylinder and the difference between the two are shown in Fig. 7 for $100 \leq R e \leq 600$. In both cases, there is a sharp decay of the agitation due to the arrest of the particle as the wake behind the sphere begins to roll, creating secondary vortices. This sudden arrest generates two different momentum exchanges with the liquid, in addition to the velocity dependent drag force. One is due to the added mass force due to the deceleration of the particle, while the second due to the unsteady viscous contribution (history force). Both exchanges are proportional to the deceleration of the particle ${ }^{42,43}$ and both are dependent on the characteristic deceleration time. ${ }^{44-46}$ This deceleration time plays a very important role in the momentum transfer between the particle and the fluid. In the simulation, the deceleration of the particle is not solved but imposed to occur on one time step. 


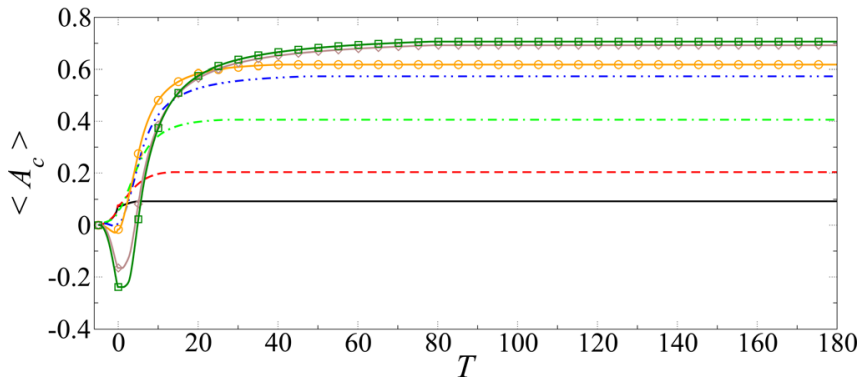

FIG. 8. (Color online) Typical curves of the average agitation index $\left\langle A_{c}\right\rangle=1 / V_{p} \int_{t_{o}}^{T} A_{c} \mathrm{~d} t$ as function of the $T$ for the collision only, showing the asymptotic behavior as function of the integration time $T$ at $50 \leq R e \leq 600$. $(-) R e=50,(--) R e=100,(-\cdot-) R e=200,(-\cdot-) R e=300,\left({ }^{\circ}-\right)$ $R e=400,(-\diamond-) \operatorname{Re}=500,(-\square-) \operatorname{Re}=600$.

To analyze the forces acting on the sphere approaching a solid wall through a thin layer would require measurements of film thickness, impact velocity, and deceleration time, where the lubrication theory applies. A detailed analysis of these contributions is outside the scope of this work, but a few remarks about the features associated with the momentum transfer are appropriate here. We consider only the ways to parameterize the agitation caused by the collision of the sphere with the wall. These parametrizations either take the form of an integral measure of the momentum exchange (related to the force on the sphere and reaction by the wall) or an integral measure of (kinetic) energy. And from this, there are two ways to define the boundaries over which these integral measures are taken (either Eulerian or Lagrangian). An integral measure of momentum is nontrivial to apply as momentum; as a concept, it is not well-defined (for unbounded flows, see Refs. 47 and 48 and references therein) and it is non-trivial to interpret (for bounded flows). Kinetic energy on the other hand is a convergent quantity (in many cases). For this reason, our approach has taken the route of assessing the kinetic energy rather than the momentum exchange.

In order to quantify the agitation produced by a single particle that can be used as boundary condition for kinetic energy on the walls, it may be useful to define an average agitation index (units of action per unit mass) as

$$
\langle A\rangle=\frac{1}{V_{p}} \int_{t_{o}}^{T} A(t) \mathrm{d} t
$$

where $V_{p}(=\pi / 6)$ is the volume of the particle, $t_{o}$ is the time the sphere begins to move, and $T$ is the time at which the fluid motion has nearly ceased. The quantity $\langle A\rangle$ approaches a constant value as $T$ approaches infinity. The average agitation index is loosely connected to the notion of "action" from classical mechanics, which postulates that the path actually followed by a physical system is that for which the action is minimized.

The asymptotic behavior of the average agitation index for the collision from Eq. (3) at different integration times $T$ is shown in Fig. 8 at $50 \leq R e \leq 600$. Notice that the average agitation index due to the collision alone $\left\langle A_{c}\right\rangle$ shown in Figure 9 is positive for all values of $R e$, which guarantees consistency with certain general properties of the Navier-Stokes equations, i.e., positiveness of the turbulent kinetic energy. ${ }^{49,50}$

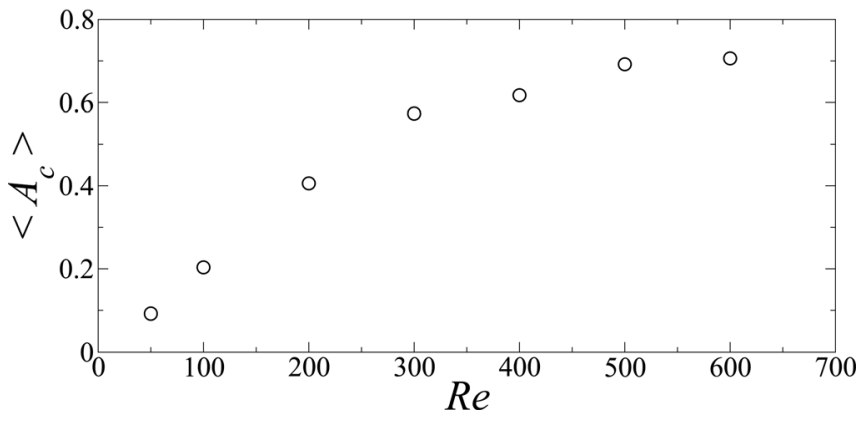

FIG. 9. Relative value of average agitation index due to the collision alone $\left\langle A_{c}\right\rangle=\left\langle A_{s}\right\rangle-\left\langle A_{e}\right\rangle$ for $50 \leq R e \leq 600$ evaluated at $T=200$.

Figure 9 suggests that $\left\langle A_{c}\right\rangle$ trends to zero for $R e \ll 1$ as inertial effects become negligible in this limit. When $R e \ll 1$, the wake behind the sphere is not created, and, as a consequence, there are not secondary vortices that could enhance the agitation of the flow. However, the effects of squeezing the fluid out of the gap between the bottom wall and the sphere, albeit small, are ubiquitous as long as there is a solid boundary at the bottom where the sphere stops. On the other hand, the effects of the bottom boundary at any $R e$ are not present in the extended cylinder, as the fluid is allowed to move through the imaginary wall.

For $R e \gg 1$, Fig. 9 indicates an asymptotic upper limit for $\left\langle A_{c}\right\rangle$. However, in this region, we should be careful when extrapolating the values of $\left\langle A_{c}\right\rangle$ to the asymptotic limit that corresponds to large $R e$, because the simulations and the experiments were performed for a range of parameters in which the flow remained axi-symmetric. It is reasonable to expect three-dimensional effects to appear as the $R e$ number is increased beyond a certain critical value, even if the flow remains in the laminar regime. If the $R e$ is further increased, the flow may become fully turbulent. Thus, the asymptotic value suggested in Fig. 9 would be invalid when the axisymmetry of the flow is broken.

\section{SUMMARY AND CONCLUSIONS}

In this investigation, we undertook detailed calculations on the fluid motion around a sphere colliding with a wall immersed in a viscous fluid. Making use of a PIV system, the agitation was obtained and compared with the numerical simulations. We found that the average agitation index $\left\langle A_{c}\right\rangle$ increases monotonically with $R e$ for the range of values considered in this investigation.

Accuracy of the modeling of particulate two-phase flows depends on how well the hydrodynamics is described. Advances in computational capabilities allow in some cases the performance of detailed simulations, but in many practical engineering applications, parts of the flow field are modelled rather than solved. ${ }^{2}$ Turbulence and particle submodels are among these models that may be used to calculate the behavior of particulate two-phase systems. In the two-phase flow coupled with engineering models (e.g., $k-\varepsilon$ turbulence model), the governing equations of fluid phase are generally described in Eulerian form, whereas the equations governing the motion of the particle can be either Eulerian or Lagrangian. In the Euler-Euler coupling model, the solid phase is 
treated as a continuum, with the disadvantage that the probabilistic characteristic of particle motion is ignored.$^{51}$ Nevertheless, in both Euler-Euler or Euler-Lagrange formulations, the wall function is usually implemented as a boundary conditions at the walls, where the turbulent kinetic energy $k$ and energy dissipation $\varepsilon$ are written in terms of the shear velocity. ${ }^{52,53}$

Here, we proposed a new integral measure that in part permits practical closure relationships near walls to be prescribed. To incorporate these detailed calculations of subgrid scale processes into under-resolved models which do not account explicitly for the particles $(k-\varepsilon$ models for example), it might be useful to use the average agitation index as a boundary condition for kinetic energy on the walls instead of the commonly used wall-function. For example, by setting $k=\left\langle A_{c}\right\rangle f$, where $f$ is the a random frequency (with Gaussian distribution) of particle collisions on the wall.

Finally, there are many aspects of integral measures applied to disperse flows which require more exploration, and this study provides the framework for further investigations. One of them is the implementation of the boundary condition at the wall in a two-phase turbulent model as suggested above and another is the motion of a sphere approaching a solid wall through a thin layer with focus on the momentum exchange due to inertia and viscous effects, which will be addressed in forthcoming papers.

\section{ACKNOWLEDGMENTS}

The comments of the anonymous Referees have greatly influenced the final version of this paper and are very much appreciated. This work was partially supported by the National Science Foundation Grant No. CBET-0608850 and by National Autonomous University of Mexico through its PAPIIT-DGAPA program (Grant No. IN 103900). A.R.-A. acknowledges the PROBETEL and IIM-UNAM for their scholarship program support. The authors acknowledge Texas Advanced Computing Center (TACC) at the University of Texas at Austin and Ira A. Fulton High Performance Computing Initiative at Arizona State University, both members of the NSF-funded Teragrid, for providing HPC and visualization resources.

${ }^{1}$ S. Nagata, Mixing: Principles and Applications (Wiley, New York, USA, 1975).

${ }^{2}$ J. C. R. Hunt, R. Delfos, I. Eames, and R. J. Perkins, "Vortices, complex flows and inertial particles," Flow, Turbul. Combust. 79, 207 (2007).

${ }^{3}$ I. Eames, "Rapidly dissolving dense bodies in an inviscid fluid," Proc. R. Soc. London, Ser. A 464, 2985 (2008).

${ }^{4}$ S.-Y. Kang, A. S. Sangani, H.-K. Tsao, and D. L. Koch, "Rheology of dense bubble suspensions," Phys. Fluids 9, 1540 (1997).

${ }^{5}$ P. D. M. Spelt and A. S. Sangani, "Properties and averaged equations for flows of bubbly liquids," Appl. Sci. Res. 58, 337 (1998).

${ }^{6} \mathrm{M}$. Brenner, "Screening mechanisms in sedimentation," Phys. Fluids 11, 754 (2001).

${ }^{7}$ G. Drazer, J. Koplik, and B. Khusid, "Microstructure and velocity fluctuations in sheared suspensions," J. Fluid Mech. 511, 237 (2004).

${ }^{8} \mathrm{C}$. E. Brennen, "A review of added mass and fluid inertial forces," Technical report, CR82.010, Nav. Civ. Eng. Lab., Dep. of the Navy, Port Hueneme, CA, (1982).

${ }^{9}$ D. D. Joseph and J. Wang, "The dissipation approximation and viscous potential flow," J. Fluid Mech. 505, 365 (2004).
${ }^{10}$ J., Magnaudet and I. Eames, "The motion of high-Reynolds-number bubbles in inhomogeneous flows," Annu. Rev. Fluid Mech. 32, 659 (2000).

${ }^{11}$ G. Riboux, F. Risso, and D. Legendre, "Experimental characterization of the agitation generated by bubbles rising at high Reynolds number," J. Fluid Mech. 643, 509 (2010).

${ }^{12}$ I. Eames, "Momentum conservation and condensing vapor bubbles," J. Heat Transfer 132, 091501 (2010).

${ }^{13}$ I. Eames and M. Gilbertson, "Mixing and drift in gas-fluidised beds," Powder Technol. 154, 185 (2005).

${ }^{14}$ R. J. Munro, N. Bethke, and S. B. Dalziel, "Sediment resuspension and erosion by vortex rings," Phys. Fluids 21, 046601 (2009).

${ }^{15}$ I. Eames and S. B. Dalziel, "Resuspension by an impacting sphere," Phys. Fluids 11, S11 (1999).

${ }^{16}$ I. Eames and S. B. Dalziel, "Dust resuspension by the flow around an impacting sphere," J. Fluid Mech. 403, 305 (2000).

${ }^{17}$ I. Eames, "Disappearing bodies and ghost vortices," Philos. Trans. R. Soc. London, Ser. A 366, 2219 (2008).

${ }^{18}$ D. Rockwell, "Vortex-body interactions," Annu. Rev. Fluid Mech. 30, 199 (1998).

${ }^{19}$ J. D. A. Walker, C. R. Smith, A. W. Cerra, and T. L. Doligalski, "The impact of a vortex ring on a wall," J. Fluid Mech. 181, 99 (1987).

${ }^{20}$ G. Joseph, R. Zenit, M. L. Hunt, and A. M. Rosenwinkel, "Particle-wall collisions in a viscous fluid," J. Fluid Mech. 433, 329 (2001).

${ }^{21} \mathrm{P}$. Gondret, M. Lance, and L. Petit, "Bouncing motion of spherical particles in fluids," Phys. Fluids 14, 643 (2002).

${ }^{22}$ R. Zenit, M. L. Hunt, and C. E. Brennen, "Collisional particle pressure measurements in solid-liquid flows," J. Fluid Mech. 353, 261 (1997).

${ }^{23} \mathrm{P}$. Orlandi and R. Verzicco, "Vortex rings impinging on walls: Axisymmetric and three-dimensional simulations," J. Fluid Mech. 256, 615 (1993).

${ }^{24}$ R. E. Lynch, J. R. Rice, and D. H. Thomas, "Tensor product analysis of partial difference equations,” Bull. Am. Math. Soc. 70, 378 (1964).

${ }^{25}$ R. Courant, K. Friedrichs, and H. Lewy, "Über die partiellen Differenzengleichungen der mathematischen Physik," Math. Ann. 100, 32 (1928).

${ }^{26} \mathrm{R}$. Verzicco and P. Orlandi, "A finite-difference scheme for three-dimensional incompressible flows in cylindrical coordinates," J. Comput. Phys. 123, 402 (1996).

${ }^{27}$ J. R. Pacheco, A. Pacheco-Vega, T. Rodić, and R. E. Peck, "Numerical simulations of heat transfer and fluid flow problems using an immersedboundary finite-volume method on non-staggered grids," Numer. Heat Transfer, Part B 48, 1 (2005).

${ }^{28}$ A. Pacheco-Vega, J. R. Pacheco, and T. Rodić, "A general scheme for the boundary conditions in convective and diffusive heat transfer with immersed boundary methods," J. Heat Transfer 129, 1506 (2007).

${ }^{29}$ G. Stringano, G. Pascazio, and R. Verzicco, "Turbulent thermal convection over grooved plates," J. Fluid Mech. 557, 307 (2006).

${ }^{30}$ S. Kang, G. Iaccarino, and F. Ham, "DNS of buoyancy-dominated turbulent flows on a bluff body using the immersed boundary method," J. Comput. Phys. 228, 3189 (2009).

${ }^{31}$ J. Kim, D. Kim, and H. Choi, "An immersed-boundary finite-volume method for simulations of flow in complex geometries," J. Comput. Phys. 171, 132 (2001).

${ }^{32}$ A. Cristallo and R. Verzicco, "Combined immersed boundary/large-eddy simulations of incompressible three dimensional complex flows," Flow Turbul. Combust. 77, 3 (2006).

${ }^{33} \mathrm{M}$. Uhlmann, "An immersed boundary method with direct forcing for the simulation of particulate flows," J. Comput. Phys. 209, 448 (2005).

${ }^{34}$ M. D. de Tullio, A. Cristallo, E. Balaras, and R. Verzicco, "Direct numerical simulation of the pulsatile flow through an aortic bileaflet mechanical heart valve," J. Fluid Mech. 622, 259 (2009).

${ }^{35}$ J. R. Pacheco, J. M. Lopez, and F. Marques, "Pinning of rotating waves to defects in finite Taylor-Couette flow," J. Fluid Mech. 666, 254 (2011).

${ }^{36}$ E. A. Fadlun, R. Verzicco, P. Orlandi, and J. Mohd-Yusof, "Combined immersed-boundary finite-difference methods for three-dimensional complex flow simulations," J. Comput. Phys. 161, 35 (2000).

${ }^{37}$ R. Verzicco, F. Lalli, and E. Campana, "Dynamics of baroclinic vortices in a rotating stratified fluid: A numerical study," Phys. Fluids 9, 419 (1997).

${ }^{38}$ S. A. Smirnov, J. R. Pacheco, and R. Verzicco, "Numerical simulations of nonlinear thermally-stratified incremental spin-up in a circular cylinder," Phys. Fluids 22, 116602 (2010).

${ }^{39}$ L. Lourenco and A. Krothapalli, "On the accuracy of velocity and vorticity measurements with PIV,” Exp. Fluids 18, 421 (1995). 
${ }^{40}$ T. Leweke, M. C. Thompson, and K. Hourigan, "Touchdown of a sphere," Phys. Fluids 16, S5 (2004).

${ }^{41}$ M. C. Thompson, T. Leweke, and K. Hourigan, "Sphere-wall collisions: Vortex dynamics and stability," J. Fluid Mech. 575, 121 (2007).

${ }^{42} \mathrm{P}$. Gondret, E. Hallouin, M. Lance, and L. Petit, "Experiments on the motion of a solid sphere toward a wall: From viscous dissipation to elastohydrodynamic bouncing," Phys. Fluids 11, 2803 (1999).

${ }^{43}$ R. Mei and C. J. Lawrence, "The flow field due to a body in impulsive motion," J. Fluid Mech. 325, 79 (1996).

${ }^{44}$ J. O. Marston, W. Yong, and S. T. Thoroddsen, "Direct verification of the lubrication force on a sphere travelling through a viscous film upon approach to a solid wall," J. Fluid Mech. 655, 515 (2010).

${ }^{45}$ A. Mongruel, C. Lamriben, S. Yahiaoui, and F. Feuillebois, "The approach of a sphere to a wall at finite Reynolds number," J. Fluid Mech. 661, 229 (2010).

${ }^{46}$ M. A. T. van Hinsberg, J. ten Thije Boonkkamp, and H. J. H. Clercx, "An efficient, second order method for the approximation of the Basset history force,” J. Comput. Phys. 230, 1465 (2011).
${ }^{47}$ B. U. Felderhof, "Transient flow caused by a sudden impulse or twist applied to a sphere immersed in a viscous incompressible fluid," Phys. Fluids 19, 073102 (2007).

${ }^{48}$ I. Eames, "Disappearing bodies and ghost vortices," Philos Trans. R. Soc. London Ser. A 366, 2219 (2008).

${ }^{49}$ C. G. Speziale, "On nonlinear $K-l$ and $K-\varepsilon$ models of turbulence," J. Fluid Mech. 178, 459 (1987).

${ }^{50}$ J. L. Lumley, "Computational modeling of turbulent flows," Adv. Appl. Mech. 18, 123 (1978).

${ }^{51}$ P. Oresta, R. Verzicco, D. Lohse, and A. Prosperetti, "Heat transfer mechanisms in bubbly Rayleigh-Bénard convection," Phys. Rev. E 80, 026304 (2009).

${ }^{52}$ Z. H. Zhao and H. J. S. Fernando, "Numerical simulation of scour around pipelines using an Euler-Euler coupled two-phase model," Environ. Fluid Mech. 7, 121 (2007).

${ }^{53}$ Z. H. Zhao and H. J. S. Fernando, "Numerical modeling of a sagging pipeline using an Eulerian two-phase model," J. Turbul. 9, 1 (2008). 